\title{
Nonuniform exponential dichotomy for block triangular sys- tems on the half line
}

\author{
Le Huy Tien, Le Duc Nhien*, Ta Van Chien
}

Department of Mathematics, Mechanics and Informatics, Vietnam National University at Hanoi, 334 Nguyen Trai, Thanh Xuan, Hanoi, Viet Nam.

\begin{abstract}
In this paper, we discuss the nonuniform exponential dichotomy properties of nonautonomous systems of linear differential equations. Since any linear differential systems are kinematically similar to a triangular system, considering the relation between the nonuniform exponential dichotomy properties of the triangular system is necessary. Without loss of generality, we consider block upper triangular systems and give the criteria for the nonuniform exponential dichotomy of triangular systems on the half line for unbounded systems.
\end{abstract}

Keywords: Nonuniform exponential dichotomy, triangular system, exponential dichotomy.

2010 MSC: 34D09, 34A30.

(C)2020 All rights reserved.

\section{Introduction}

Stability and dichotomy are well-studied topics in theory of differential equations. In particular, it is central in the construction of topological conjugacies, invariant manifolds, and normal forms. In finite dimensional Euclidean spaces, a linear differential equation

$$
\dot{x}=A(t) x,
$$

on the half line $J=\mathbb{R}_{+}$or $\mathbb{R}_{-}$is exponentially dichotomous if there are projection $\mathrm{P}(\mathrm{t}): \mathbb{R}^{\mathrm{n}} \rightarrow \mathbb{R}^{\mathrm{n}}$ and positive constants $K>1, \alpha>0$ such that with $t, s \in J$,

$$
T(t, s) P(s)=P(t) T(t, s),
$$

and

$$
\|T(t, s) P(s)\| \leqslant K e^{-\alpha(t-s)} \quad \text { for } t \geqslant s, \quad\|T(t, s)(I-P(s))\| \leqslant K e^{-\beta(s-t)} \quad \text { for } t \leqslant s,
$$

\footnotetext{
${ }^{*}$ Corresponding author

Email addresses: tienlh@viasm.edu.vn (Le Huy Tien), nhien0610@gmail.com (Le Duc Nhien), tavanchien_s16@hus.edu.vn (Ta Van Chien)
}

doi: $10.22436 /$ jnsa.013.02.02

Received: 2019-05-30 Revised: 2019-08-06 Accepted: 2019-08-26 
where $T(t, s)$ denotes the evolution operator of Eq. (1.1). Roughly speaking, an exponentially dichotomous system can be invariantly decomposed into two subsystems and dynamics on subsystems are stable in forward and backward directions of time.

There are a lot of efforts to extend this concept to systems with nonuniformity. The nonuniformity means that $\mathrm{K}$ is a function which depend on the initial time. The implication of such extension was indicated by Perron (see [11], pp. 705-706) early in 1930 for asymptotical stability. Coppel (see [10], p. 12) also showed that a planar nonautonomous systems without bounded growth may not have an exponential dichotomy even if it is a uniform contraction (respectively, expansion) on the stable (respectively, unstable) subspace. The formal definitions of the case of nonuniformity was given by Barreira and Valls (see $[5,8])$. The requirement of uniform stability or uniform conditional stability is often too stringent for the dynamics. In particular, the notion of a nonuniform exponential dichotomy is much more typical, particularly, in the context of ergodic theory. For example, almost all trajectories (and in fact all Lyapunov regular trajectories) of a diffeomorphism or a flow with nonzero Lyapunov exponents have a nonuniform exponential dichotomy (see $[3,4,6])$. Besides, the relations between the nonuniform exponential dichotomies and Fredholm operators are studied as in $[1,2]$.

Because of the essential role of the concept of a nonuniform exponential dichotomy, it is important to have helpful characterizations. Our main purpose is giving the relations between the nonuniform exponential dichotomy properties of the triangular system and its diagonal part. Particularly, we show that if a block upper triangular system has a nonuniform exponential dichotomy on a half line, then its diagonal part also has. When the off diagonal part is bounded in term of a family $\left\{\|\cdot\|_{t}\right\}_{t \in J}$ of norms (see a below concept), then the converse holds. The other characterizations of nonuniform exponential dichotomy in terms of admissibility can be seen in $[7,12]$.

\section{Preliminaries and main results}

With the notation in Section 1, we say that Eq. (1.1) has a nonuniform exponential dichotomy if there exist a projection $\mathrm{P}(\mathrm{t}): \mathbb{R}^{\mathrm{n}} \rightarrow \mathbb{R}^{\mathrm{n}}$ satisfying (1.2), constant $\alpha>0$, and a continuous function $\mathrm{K}: \mathrm{J} \rightarrow[1,+\infty)$ such that

$$
\|T(t, s) P(s)\| \leqslant K(s) e^{-\alpha(t-s)} \quad \text { for } t \geqslant s, \quad\|T(t, s)(I-P(s))\| \leqslant K(s) e^{-\beta(s-t)} \quad \text { for } t \leqslant s,
$$

for all $s, t \in J$.

Consider the block upper triangular system

$$
\dot{x}=\left(\begin{array}{cc}
A(t) & C(t) \\
0 & B(t)
\end{array}\right) x
$$

with $x \in \mathbb{R}^{\mathrm{d}}, \mathrm{y} \in \mathbb{R}^{\mathrm{n}-\mathrm{d}}, 1 \leqslant \mathrm{~d} \leqslant \mathrm{n}$. In the following result, one show that if a not necessarily bounded block triangular system has an exponential dichotomy on a half line, then so does block diagonal part.

Theorem 2.1. Assume that (2.1) has a nonuniform exponential dichotomy with bound $\mathrm{K}(\mathrm{s})$ and exponents $\alpha, \beta$. Then both linear systems $\dot{x}=\mathrm{A}(\mathrm{t}) \mathrm{x}$ and $\dot{\mathrm{y}}=\mathrm{B}(\mathrm{t}) \mathrm{y}$ have a nonuniform exponential dichotomy on $\mathrm{J}$ with the same bound and exponents.

Unfortunately, we do not know if the converse of Theorem 2.1 holds, namely, if a block diagonal part has a nonuniform exponential dichotomy then a block upper triangular system has. The next comments give some light to this question.

For a given family $\left\{\|\cdot\|_{t}\right\}_{t \in J}$ of norms, we use $\left(\mathbb{R}^{n},\|\cdot\|_{t}\right)$ to denote the space $\mathbb{R}^{n}$ with norm $\|\cdot\|_{t}$ for a fixed $t \in J$ and $\|\cdot\|_{s t}$, to denote the norm of linear operator from $\left(\mathbb{R}^{n},\|\cdot\|_{s}\right)$ to $\left(\mathbb{R}^{n},\|\cdot\|_{t}\right)$ for each $t, s \in J$. Since every two norms of $\mathbb{R}^{n}$ are mutually equivalent, for a given family $\left\{\|\cdot\|_{t}\right\}_{t \in J}$ of norms there exist two functions $L_{1}, L_{2}: J \rightarrow(0,+\infty)$ such that

$$
\mathrm{L}_{1}(\mathrm{t})\|x\| \leqslant\|x\|_{\mathrm{t}} \leqslant \mathrm{L}_{2}(\mathrm{t})\|x\|, \quad x \in X, \mathrm{t} \in \mathrm{J} .
$$


We call that a family $\left\{\|\cdot\|_{t}\right\}_{t \in J}$ of norms is a continuous family of norms if the mapping $t \rightarrow\|x\|_{t}$ is continuous on $J$ for each fixed $x \in \mathbb{R}^{n}$. Furthermore, if family $\left\{\|\cdot\|_{t}\right\}_{t \in J}$ is continuous then there exist two positive continuous functions $L_{1}$ and $L_{2}$ such that (2.2) holds. A continuous family of norms is said to have a uniform lower bound (or uniform upper bound) if $\mathrm{L}_{1}$ (or $\mathrm{L}_{2}$ ) given in (2.2) is a positive constant. A continuous family of norms is said to have uniform bounds if $L_{1}$ and $L_{2}$ are both positive constants.

Our approach to proving main theorems is based on the following lemma about uniformization ([12, Lemma 1]).

Lemma 2.2. Eq. (1.1) has a nonuniform exponential dichotomy on $\mathrm{J}$ if and only if there exists a continuous family $\left\{\|\cdot\|_{\mathrm{t}}\right\}_{\mathrm{t} \in \mathrm{J}}$ of norms with a uniform lower bound such that Eq. (1.1) has a uniform exponential dichotomy with respect to $\left\{\|\cdot\|_{t}\right\}_{t \in J}$, i.e., there are a projection $\mathrm{P}(\mathrm{t}): \mathbb{R}^{\mathrm{n}} \rightarrow \mathbb{R}^{\mathrm{n}}$ and constants $\alpha>0$ and $\mathrm{K}>1$ such that the invariant decomposition condition (1.2) is satisfied and the following estimates hold

$$
\|\mathrm{T}(\mathrm{t}, \mathrm{s}) \mathrm{P}(\mathrm{s})\|_{s \mathrm{t}} \leqslant K \mathrm{e}^{-\alpha(\mathrm{t}-\mathrm{s})} \quad \text { for } \mathrm{t} \geqslant \mathrm{s}, \quad\|\mathrm{T}(\mathrm{t}, \mathrm{s})(\mathrm{I}-\mathrm{P}(\mathrm{s}))\|_{s \mathrm{t}} \leqslant \mathrm{K} \mathrm{e}^{-\beta(s-\mathrm{t})} \quad \text { for } \mathrm{t} \leqslant \mathrm{s},
$$

where $\mathrm{t}, \mathrm{s} \in \mathrm{J}$.

With this lemma we obtain the following result.

Theorem 2.3. If $\mathrm{C}(\mathrm{t}) \in \mathrm{C}_{\mathrm{b}}^{0}\left(\mathrm{~J}, \sup _{\mathrm{t} \in \mathrm{J}}\|\cdot\|_{\mathrm{t}}\right)$ and $\dot{\mathrm{x}}=\mathrm{A}(\mathrm{t}) \mathrm{x}, \dot{\mathrm{y}}=\mathrm{B}(\mathrm{t}) \mathrm{y}$ have a nonuniform exponential dichotomy on J. Then

$$
\dot{x}=\left(\begin{array}{cc}
A(t) & C(t) \\
0 & B(t)
\end{array}\right) x
$$

also have a nonuniform exponential dichotomy on $\mathrm{J}$.

The result above for the uniform exponential dichotomy is given by Battelli and Palmer (see [9]).

\section{Proof of main theorems}

Proof of Theorem 2.1. Let $V_{1} \subset \mathbb{R}^{d}$ be the subspace of initial conditions at $(t=0)$ for which the solution $\dot{x}=A(t) x$ is bounded on J and takes $V_{2}$ as any fixed complement of $V_{1}$ in $\mathbb{R}^{d}$.

Next let $W_{1} \subset \mathbb{R}^{n-d}$ be the subspace of initial conditions $\eta$ at $(t=0)$ for which $Y(t, 0) \eta$ is bounded on $J(Y(t, 0) \eta$ is a solution of $\dot{x}=B(t) x)$ and the equation

$$
\dot{x}=A(t) x+C(t) Y(t, 0) \eta,
$$

has a bounded solution on $J$ and takes any fixed complement $W_{2}$ of $W_{1}$ in $\mathbb{R}^{n-d}$.

Let $x_{b}(t)$ be a bounded solution of (3.1) for which $x_{b}(0) \in V_{2}$. Using constants variation, we have a solution of (3.1) that is

$$
x_{b}(t)=X(t, 0) x_{b}(0)+\int_{0}^{t} X(t, s) C(s) Y(s, 0) \eta d s .
$$

Suppose there exist two initial conditions $\eta_{1}, \eta_{2} \in V_{2}$ in which solutions of (3.1) are bounded, we have

$$
x_{1}(t)=X(t, 0) \eta_{1}+\int_{0}^{t} X(t, s) c(s) Y(s, 0) \eta d s, \quad x_{2}(t)=X(t, 0) \eta_{2}+\int_{0}^{t} X(t, s) c(s) Y(s, 0) \eta d s
$$

Then

$$
x_{1}(t)-x_{2}(t)=X(t, 0)\left(\eta_{1}-\eta_{2}\right) .
$$

Because $x_{1}(t), x_{2}(t)$ are bounded, we have

$$
X(t, 0)\left(\eta_{1}-\eta_{2}\right) \text { is bounded. }
$$


Thus, $\eta_{1}-\eta_{2} \in V_{1}$, on the other side $V_{1} \cap V_{2}=\{0\}$, and $\eta_{1}-\eta_{2} \in V_{1}$, then, $\eta_{1}=\eta_{2}$. Thus, $x_{b}(t)$ is a unique bounded solution of (3.1) for which $x_{b}(0) \in V_{2}$.

We consider the linking operator

$$
\mathrm{L}: \mathrm{W}_{1} \rightarrow \mathrm{V}_{2}, \quad \eta \rightarrow \mathrm{x}_{\mathrm{b}}(0)
$$

We have $\mathrm{L}_{1}=x_{\mathrm{b}_{1}}(0), \mathrm{L} \eta_{2}=x_{\mathrm{b}_{2}}(0)$, then

$$
\mathrm{L} \eta_{1}+\mathrm{L} \eta_{2}=x_{\mathrm{b}_{1}}(0)+\mathrm{x}_{\mathrm{b}_{2}}(0)
$$

One sets

$$
x_{b}(t)=X(t, 0)\left[x_{b_{1}}(0)+x_{b_{2}}(0)\right]+\int_{0}^{t} X(t, s) C(s) Y(s, 0)\left(\eta_{1}+\eta_{2}\right) d s=x_{b_{1}}(t)+x_{b_{2}}(t) \text { is bounded. }
$$

Explicit $x_{b}(t)$ is the bounded solution of (3.1) and $x_{b}(0)=x_{b_{1}}(0)+x_{b_{2}}(0) \in V_{2}$, and $x_{b}(t)$ is a unique bounded solution of (3.1). Then

$$
\mathrm{L}\left(\eta_{1}+\eta_{2}\right)=\mathrm{x}_{\mathrm{b}_{1}}(0)+\mathrm{x}_{\mathrm{b}_{2}}(0)=\mathrm{L}_{1}+\mathrm{L} \eta_{2},
$$

we get $\mathrm{L}$ as a linear operator.

We take $(\xi, \eta)$ as the initial conditions of the bounded solution of (2.1), then $\eta \in W_{1}$ and $\xi=\xi_{0}+L \eta$ with $\xi_{0} \in V_{1}$. Because $(\xi, \eta)$ are the initial conditions of the bounded solution and

$$
x(t)=X(t, 0) \xi+\int_{0}^{t} X(t, s) C(s) Y(s, 0) \eta d s \text { is bounded, }
$$

it is mandatory that $\xi, \eta$ must satisfy $Y(t, 0) \eta$ as bounded. We have

$$
x_{1}(t)=X(t, 0) \xi+\int_{0}^{t} X(t, s) C(s) Y(s, 0) \eta d s \text { is bounded. }
$$

That means $\eta \in W_{1}$,

$$
x_{2}(t)=X(t, 0) L \eta+\int_{0}^{t} X(t, s) C(s) Y(s, 0) \eta d s \text { is bounded. }
$$

Combining (3.2) and (3.3), we have $x_{1}(t)-x_{2}(t)=X(t, 0)(\xi-L \eta)$. On the other hand, $x_{1}(t)-x_{2}(t)$ is bounded, so $\xi-\mathrm{L} \eta=\xi_{0} \in \mathrm{V}_{1}$.

Next when $J=\mathbb{R}_{+}$, let $P^{A}: \mathbb{R}^{d} \rightarrow \mathbb{R}^{d}$ be the projection such that Range $\left(P^{A}\right)=V_{1}, \operatorname{Ker}^{A}=V_{2}$ and $\mathrm{Q}: \mathbb{R}^{\mathrm{n}-\mathrm{d}} \rightarrow \mathbb{R}^{\mathrm{n}-\mathrm{d}}$ be the projection such that $\operatorname{Range}(\mathrm{Q})=\mathrm{W}_{1}, \operatorname{Ker}(\mathrm{Q})=\mathrm{W}_{2}$. When $J=\mathbb{R}_{-}$, we switch the range and null space in both projections. Then if we define the projection $\mathrm{P}$ as,

$$
\left(\begin{array}{cc}
\mathrm{P}^{\mathrm{A}} & \mathrm{LQ} \\
0 & \mathrm{Q}
\end{array}\right)\left(J=\mathbb{R}_{+}\right),\left(\begin{array}{cc}
\mathrm{P}^{\mathrm{A}} & \mathrm{L}\left(\mathrm{I}_{\mathrm{n}-\mathrm{d}}-\mathrm{Q}\right) \\
0 & \mathrm{Q}
\end{array}\right)\left(\mathrm{J}=\mathbb{R}_{-}\right),
$$

we see that the range of $P$ when $J=\mathbb{R}_{+}$(the null space when $J=\mathbb{R}_{-}$) consists of all vectors $\left(\xi_{0}+L \eta, \eta\right)$ for some $\xi_{0} \in V_{1}$ and $\eta \in W_{1}$ and thus coincides with the subspace of initial values of the solutions of (2.1) bounded on J.

Consider the equations,

$$
\begin{aligned}
& \dot{x}=A(t) x+C(t) y \\
& \dot{y}=B(t) y .
\end{aligned}
$$


We have $y(t)=Y(t, 0) \eta$ as the solution of (3.6), substituting (3.5), we obtain,

$$
\dot{x}=A(t) x+C(t) Y(t, 0) \eta
$$

and

$$
x(t)=X(t, 0) \xi+\int_{0}^{t} X(t, s) C(s) Y(s, 0) \eta d s .
$$

We have, the transition matrix of (2.1) as

$$
T(t, s)=\left(\begin{array}{cc}
X(t, s) & \int_{s}^{t} X(t, \tau) C(\tau) Y(\tau, s) d \tau \\
0 & Y(t, s)
\end{array}\right) .
$$

We set

$$
W(t, s)=\int_{s}^{t} X(t, \tau) C(\tau) Y(\tau, s) d \tau
$$

then,

$$
\begin{aligned}
& P(t)=T(t, 0) P T(0, t)=\left(\begin{array}{cc}
X(t, 0) & W(t, 0) \\
0 & Y(t, 0)
\end{array}\right)\left(\begin{array}{cc}
P^{A} & L Q \\
0 & Q
\end{array}\right)\left(\begin{array}{cc}
X(t, 0) & W(0, t) \\
0 & Y(0, t)
\end{array}\right) \\
& =\left(\begin{array}{cc}
X(t, 0) P^{A} X(0, t) & * \\
0 & Y(t, 0) Q Y(0, t)
\end{array}\right) .
\end{aligned}
$$

We see that for any $\xi \in \mathbb{R}^{\mathrm{d}}$ and $s \in \mathrm{J}$, we have

$$
\mathrm{P}(\mathrm{s})\left(\begin{array}{l}
\xi \\
0
\end{array}\right)=\left(\begin{array}{l}
\mathrm{P}^{\mathrm{A}}(\mathrm{s}) \xi \\
0
\end{array}\right)
$$

where $P^{A}(s)=X(t, 0) P^{A} X(0, t)$, and

$$
\left(I_{n}-P(s)\right)\left(\begin{array}{l}
\xi \\
0
\end{array}\right)=\left(\begin{array}{c}
\left(I_{d}-P^{A}(s)\right) \xi \\
0
\end{array}\right) .
$$

As a consequence $\left\|P^{A}(s)\right\| \leqslant\|P(s)\|$ and, for $\xi \in \mathbb{R}^{d}$ and $s \leqslant t$ in $J$, we get

$$
\left\|X(t, s) P^{A}(s) \xi\right\|=\left\|T(t, s)\left(\begin{array}{l}
P^{A}(s) \xi \\
0
\end{array}\right)\right\|=\left\|T(t, s) P(s)\left(\begin{array}{l}
\xi \\
0
\end{array}\right)\right\| \leqslant K(s) e^{-\alpha(t-s)}\|\xi\|
$$

and, similarly, for any $\xi \in \mathbb{R}^{\mathrm{d}}$ and $\mathrm{t} \leqslant \mathrm{s}$, in $\mathrm{J}$

$$
\left\|X(t, s)\left(I_{d}-P^{A}(s)\right) \xi\right\|\left\|T(t, s)\left(\begin{array}{l}
\left(I_{d}-P^{A}(s)\right) \xi \\
0
\end{array}\right)\right\|=\left\|T(t, s)\left(I_{n}-P(s)\right)\left(\begin{array}{l}
\xi \\
0
\end{array}\right)\right\| \leqslant K(s) e^{\beta(t-s)}\|\xi\| .
$$

This completes the proof of the nonuniform exponential dichotomy on $J$ of the linear system $\dot{x}=A(t) x$ with projection $\mathrm{P}^{\mathrm{A}}$.

Next, we prove that $\dot{y}=B(t) y$ has a nonuniform exponential dichotomy on J. To this end, consider the adjoint system

$$
\left\{\begin{array}{l}
\dot{x}=-A^{*}(t) x \\
\dot{y}=-C^{*}(t) x-B^{*}(t) y
\end{array}\right.
$$

which is block lower triangular but can be considered as the block upper triangular system

$$
\left\{\begin{array}{l}
\dot{u}=-C^{*}(t) v-B^{*}(t) u \\
\dot{v}=-A^{*}(t) v
\end{array}\right.
$$


with $u=y$ and $v=x$. The lower triangular system (3.7) has a nonuniform exponential dichotomy on $J$ with projection $I_{n}-P^{*}$, bound $K(s)$, and exponents $\alpha, \beta$ where $P$ is as in (3.4), and so (3.8) has a nonuniform exponential dichotomy on $\mathrm{J}$ with projections

$$
\left(\begin{array}{cc}
\mathrm{I}_{\mathrm{n}-\mathrm{d}}-\mathrm{Q}^{*} & -\mathrm{Q}^{*} \mathrm{~L}^{*} \\
0 & \mathrm{I}_{\mathrm{d}}-\left(\mathrm{P}^{A}\right)^{*}
\end{array}\right),\left(\begin{array}{cc}
\mathrm{I}_{\mathrm{n}-\mathrm{d}}-\mathrm{Q}^{*} & \left(\mathrm{I}_{\mathrm{n}-\mathrm{d}}-\mathrm{Q}^{*}\right) \mathrm{L}^{*} \\
0 & \mathrm{I}_{\mathrm{d}}-\left(\mathrm{P}^{A}\right)^{*}
\end{array}\right)
$$

respectively for $J=\mathbb{R}_{+}$and $J=\mathbb{R}_{-}$, and with bound $K(s)$ and exponents $\alpha, \beta$. Since this projection is in block upper triangular form, it is as in (3.4) that $\dot{y}=-B^{*}(t) y$ has a nonuniform exponential dichotomy on $J$ with projection $I_{n-d}-Q^{*}$ and the same bound and exponents, and hence so does $\dot{y}=B(t) y$ with $Q$ as the projection. Therefore, we may take $\mathrm{P}^{\mathrm{B}}=\mathrm{Q}$, which indicates that $\mathrm{W}_{1}=\operatorname{Range}\left(\mathrm{P}^{\mathrm{B}}\right)$ and that $\mathrm{P}$ has the form (3.4) as stated in the theorem.

Here, we derive the rank conditions. With $\mathrm{P}$ as defined in the statement of the theorem, we observe that $(\xi, \eta) \in \operatorname{Ker}(P)$ if and only if $P^{A} \xi=0$ and $P^{B} \eta=0$ so that $\operatorname{dim} \operatorname{Ker}(P)=\operatorname{dim} \operatorname{Ker}\left(P^{A}\right)+\operatorname{dim} \operatorname{Ker}(Q)$. Hence, $\operatorname{rank}(\mathrm{P})=\operatorname{rank}(\mathrm{P})^{\mathrm{A}}+\operatorname{rank}\left(\mathrm{P}^{\mathrm{B}}\right)$ follows, thus completing the proof of the theorem.

Remark 3.1. In particular we have proved that if the block upper triangular system (2.1) has a nonuinform exponential dichotomy on $J$, then for any bounded solution $y(t)$ of $\dot{y}=B(t) y$ there exists a bounded solution of the linear inhomogeneous system $\dot{x}=A(t) x+C(t) y(t)$ and this holds even if $C(t)$ is not bounded. However the fact that the upper triangular system has a nonuniform exponential dichotomy imposes some restrictions on the choice of $C(t)$.

Remark 3.2. We can derive formulas for the linking operators. From the proof of Theorem 2.1 we see that for any $\eta \in \operatorname{Range}\left(\mathrm{P}^{\mathrm{B}}\right)$, the system

$$
\dot{x}=A(t) x+C(t) Y(t, 0) \eta
$$

has a unique solution $x_{b}(t)$ bounded on J such that $P^{A} x_{b}(0)=0$. By standard methods it is proved that when $\mathrm{J}=\mathbb{R}_{+}$,

$$
x_{b}(t)=\int_{0}^{t} X(t, s) P^{A}(s) C(s) Y(s, 0) \eta d s-\int_{t}^{+\infty} X(t, s)\left(I_{d}-P^{A}(s)\right) C(s) Y(s, 0) \eta d s
$$

Hence

$$
x_{b}(0)=-\int_{0}^{+\infty} X(0, s)\left(I_{d}-P^{A}(s)\right) C(s) Y(s, 0) \eta d s,
$$

and

$$
\mathrm{P}^{\mathrm{A}}\left(\mathrm{x}_{\mathrm{b}}(0)\right)=-\int_{0}^{+\infty} \mathrm{X}(0, \mathrm{~s})\left(\mathrm{P}^{\mathrm{A}}(\mathrm{s})-\left(\mathrm{P}^{\mathrm{A}}\right)^{2}\right) \mathrm{C}(\mathrm{s}) \mathrm{Y}(\mathrm{s}, 0) \eta \mathrm{d} \mathrm{s}=0 .
$$

And so the linking operator on $\mathbb{R}_{+}$is given by

$$
\mathrm{L} \eta=x_{\mathrm{b}}(0)=-\int_{0}^{+\infty} X(0, s)\left(I_{d}-P^{A}(s)\right) C(s) Y(s, 0) \eta d s .
$$

When $\mathrm{J}=\mathbb{R}_{-}$,

$$
x_{b}(t)=\int_{-\infty}^{t} X(t, s) P^{A}(s) C(s) Y(s, 0) \eta d s-\int_{t}^{0} X(t, s)\left(I_{d}-P^{A}(s)\right) C(s) Y(s, 0) \eta d s
$$


and then the linking operator on $\mathbb{R}_{-}$is given by

$$
L \eta=\int_{-\infty}^{0} X(0, s) P^{A}(s) C(s) Y(s, 0) \eta d s
$$

Proof of Theorem 2.3. Considering $J=\mathbb{R}_{+}$, we have the transition matrix (2.1) is

$$
T(t, s)=\left(\begin{array}{cc}
X(t, s) & W(t, s) \\
0 & Y(t, s)
\end{array}\right)
$$

where $X(t, s), Y(t, s)$ are the transition matrices of $\dot{x}=A(t) x$ and $\dot{y}=B(t) y$, respectively, and

$$
W(t, s)=\int_{s}^{t} X(t, \tau) C(\tau) Y(\tau, s) d \tau
$$

If system (2.1) has a nonuniform exponential dichotomy on J, then Theorem 2.1 tells us we can take the projection $P$ to be in the form of (3.4), and the linking operator $L$ is defined as follows

$$
\mathrm{L} \eta=-\int_{0}^{+\infty}\left(\mathrm{I}_{\mathrm{d}}-\mathrm{P}^{\mathrm{A}}\right) X(0, \mathrm{t}) \mathrm{C}(\mathrm{t}) \mathrm{Y}(\mathrm{t}, 0) \eta \mathrm{dt}, \quad\left(\eta \in \text { Range }\left(\mathrm{P}^{\mathrm{B}}\right)\right) \text {. }
$$

Note that

$P(t)=T(t, 0) P T(0, t)=\left(\begin{array}{cc}X(t, 0) & W(t, 0) \\ 0 & Y(t, 0)\end{array}\right)\left(\begin{array}{cc}P^{A} & L P^{B} \\ 0 & P^{B}\end{array}\right)\left(\begin{array}{cc}X(0, t) & W(0, t) \\ 0 & Y(0, t)\end{array}\right)=\left(\begin{array}{cc}P^{A}(t) & R(t) \\ 0 & P^{B}(t)\end{array}\right)$, where $P^{A}(t)=X(t, 0) P^{A} X(0, t), P^{B}(t)=Y(t, 0) P^{B} Y(0, t)$ and $R(t)$ solves

$$
\dot{R}=A(t) R-R B(t)+C(t) P^{B}(t)-P^{A}(t) C(t)
$$

with $R(0)=L P^{B}$. By variation of constants

$$
R(t)=X(t, 0) R(0) Y(0, t)+\int_{0}^{t} X(t, \tau)\left[C(\tau) P^{B}(\tau)-P^{A}(\tau) C(\tau)\right] Y(\tau, t) d \tau .
$$

Then, from the definition of $\mathrm{L}$, we have

$$
L P^{B}=-\int_{0}^{+\infty}\left(I_{d}-P^{A}(\tau)\right) X(0, \tau) C(\tau) Y(\tau, 0) P^{B} d \tau
$$

Hence

$$
\begin{aligned}
R(t)= & -\int_{t}^{+\infty} X(t, \tau)\left(I_{d}-P^{A}(\tau)\right) C(\tau) P^{B}(\tau) Y(\tau, t) d \tau \\
& -\int_{0}^{t} X(t, \tau) P^{A}(\tau) C(\tau)\left[I_{n-d}-P^{B}(\tau)\right] Y(\tau, t) d \tau .
\end{aligned}
$$

We now prove that (2.1) has a nonuniform exponential dichotomy on J. First we prove that

$$
\|T(t, s) P(s)\| \leqslant K(s) e^{-\alpha(t-s)} \text { for } s \leqslant t \text { in } J .
$$


We have

$$
T(t, s) P(s)=\left(\begin{array}{cc}
X(t, s) P^{A}(s) & W(t, s) P^{B}(s)+X(t, s) R(s) \\
0 & Y(t, s) P^{B}(s)
\end{array}\right) .
$$

Note that we can write

$$
\begin{aligned}
W(t, s) P^{B}(s) & =\int_{s}^{t} X(t, \tau) C(\tau) P^{B}(\tau) Y(\tau, s) d \tau \\
& =\int_{s}^{t} X(t, \tau)\left(I_{d}-P^{A}(\tau)\right) C(\tau) P^{B}(\tau) Y(\tau, s) d \tau+\int_{s}^{t} X(t, \tau) P^{A}(\tau) C(\tau) P^{B}(\tau) Y(\tau, s) d \tau,
\end{aligned}
$$

and

$$
\begin{aligned}
X(t, s) R(s)= & X(t, s)\left[-\int_{s}^{+\infty} X(s, \tau)\left(I_{d}-P^{A}(\tau)\right) C(\tau) P^{B}(\tau) Y(\tau, s) d \tau\right. \\
& \left.-\int_{0}^{s} X(s, \tau) P^{A}(\tau) C(\tau)\left[I_{n-d}-P^{B}(\tau)\right] Y(\tau, s) d \tau\right] \\
= & -\int_{s}^{+\infty} X(t, \tau)\left(I_{d}-P^{A}(\tau)\right) C(\tau) P^{B}(\tau) Y(\tau, s) d \tau \\
& -\int_{0}^{s} X(t, \tau) P^{A}(\tau) C(\tau)\left[I_{n-d}-P^{B}(\tau)\right] Y(\tau, s) d \tau .
\end{aligned}
$$

Hence

$$
\begin{aligned}
W(t, s) P^{B}(s)+X(t, s) R(s) & =\int_{s}^{t} X(t, \tau) P^{A}(\tau) C(\tau) P^{B}(\tau) Y(\tau, s) d \tau \\
& -\int_{t}^{+\infty} X(t, \tau)\left[I_{d}-P^{A}(\tau)\right] C(\tau) P^{B}(\tau) Y(\tau, s) d \tau \\
& -\int_{0}^{s} X(t, \tau) P^{A}(\tau) C(\tau)\left[I_{n-d}-P^{B}(\tau)\right] Y(\tau, s) d \tau .
\end{aligned}
$$

With $s \leqslant t, \in J$ and $C(t) \in C_{b}^{0}\left(J, \sup _{t \in J}\|.\|_{t}\right)$, we have

$$
\begin{aligned}
\left\|W(t, s) P^{B}(s)+X(t, s) R(s)\right\| \leqslant & \left\|W(t, s) P^{B}(s)+X(t, s) R(s)\right\|_{t} \\
\leqslant & \int_{s}^{t}\left\|X(t, \tau) P^{A}(\tau)\right\|\left\|_{s t}\right\| C(\tau)\left\|_{s}\right\| P^{B}(\tau) Y(\tau, s) \|_{s t} d \tau \\
& +\int_{t}^{+\infty}\left\|X(t, \tau)\left(I_{d}-P^{A}(\tau)\right)\right\|_{s t}\|C(\tau)\|_{s}\left\|P^{B}(\tau) Y(\tau, s)\right\|_{s t} d \tau \\
& +\int_{0}^{s}\left\|X(t, \tau) P^{A}(\tau)\right\|_{s t}\|C(\tau)\|_{s}\left\|\left(I_{n-d}-P^{B}(\tau)\right) Y(\tau, s)\right\|_{s t} d \tau
\end{aligned}
$$




$$
\begin{aligned}
& \leqslant k_{1} k_{2}\|C\|_{t}\left[\frac{e^{-\alpha_{2}(t-s)}-e^{-\alpha_{1}(t-s)}}{\alpha_{1}-\alpha_{2}}+\frac{e^{-\alpha_{2}(t-s)}}{\alpha_{2}+\beta_{1}}+\frac{e^{-\alpha_{1}(t-s)}}{\alpha_{1}+\beta_{2}}\right] \\
& \leqslant k_{1} k_{2}\|C\|_{t}\left[\frac{1}{\left|\alpha_{1}-\alpha_{2}\right|}+\frac{1}{\alpha_{2}+\beta_{1}}+\frac{1}{\alpha_{1}+\beta_{2}}\right] e^{-\alpha(t-s)} .
\end{aligned}
$$

Where, exponents $\alpha_{1}, \alpha_{2}, \beta_{1}, \beta_{2}>0$ and constants $k_{1}, k_{2} \geqslant 1, \alpha=\min \left\{\alpha_{1}, \alpha_{2}\right\}$. If $\alpha_{1}=\alpha_{2}$, we have

$$
\int_{s}^{t} e^{-\alpha_{1}(t-\tau)} e^{-\alpha_{1}(\tau-s)} d \tau=(t-s) e^{-\alpha_{1}(t-s)} \leqslant c_{\alpha} e^{-\alpha(t-s)},
$$

so that $\frac{1}{\left|\alpha_{1}-\alpha_{2}\right|}$ in the last inequality is replaced by $c_{\alpha}$. Furthermore, note that $t=s$, we do not have the first of the three terms in the inequality above so that we get

$$
\|R(t)\| \leqslant k_{1} k_{2}\|C\|_{t}\left[\frac{1}{\alpha_{2}+\beta_{1}}+\frac{1}{\alpha_{1}+\beta_{2}}\right] .
$$

Next, we prove that

$$
\left\|\mathrm{T}(\mathrm{t}, \mathrm{s})\left[\mathrm{I}_{\mathrm{n}}-\mathrm{P}(\mathrm{s})\right]\right\| \leqslant \mathrm{K}(\mathrm{s}) \mathrm{e}^{-\beta(s-\mathrm{t})} \text { for } \mathrm{t} \leqslant \mathrm{s} \text { in } \mathrm{J} .
$$

Here, $T(t, s)\left[I_{n}-P(s)\right]=\left[I_{n}-P(t)\right] T(t, s)$, which equals

$$
\left(\begin{array}{cc}
\left(I_{d}-P^{A}(t)\right) X(t, s) & \left(I_{d}-P^{A}(t)\right) W(t, s)-R(t) Y(t, s) \\
0 & \left(I_{n-d}-P^{B}(t)\right) Y(t, s)
\end{array}\right) .
$$

Now, when $\mathrm{J}=\mathbb{R}_{+}$, we have

$$
\begin{aligned}
R(t) Y(t, s)= & -\int_{0}^{t} X(t, \tau) P^{A}(\tau) C(\tau)\left(I_{n-d}-P^{B}(\tau)\right) Y(\tau, s) d \tau \\
& -\int_{t}^{+\infty} X(t, \tau)\left(I_{d}-P^{A}(\tau)\right) C(\tau) P^{B}(\tau) Y(\tau, s) d \tau
\end{aligned}
$$

and

$$
\left(I_{d}-P^{A}(t)\right) W(t, s)=\int_{s}^{t} X(t, \tau)\left(I_{d}-P^{A}(\tau)\right) C(\tau) Y(\tau, s) d \tau .
$$

So, after some rearrangements

$$
\begin{aligned}
\left(I_{d}-P^{A}(t)\right) W(t, s)-R(t) Y(t, s)= & \int_{s}^{+\infty} X(t, \tau)\left(I_{d}-P^{A}(\tau)\right) C(\tau) P^{B}(\tau) Y(\tau, s) d \tau \\
& -\int_{t}^{s} X(t, \tau)\left(I_{d}-P^{A}(\tau)\right) C(\tau)\left(I_{n-d}-P^{B}(\tau)\right) Y(\tau, s) d \tau \\
& +\int_{0}^{t} X(t, \tau) P^{A}(\tau) C(\tau)\left(I_{n-d}-P^{B}(\tau)\right) Y(\tau, s) d \tau .
\end{aligned}
$$


Then, $t \leqslant s ; t, s \in J$ and $C(t) \in C_{b}^{0}\left(J, \sup _{t \in J}\|.\|_{t}\right)$, we have

$$
\begin{aligned}
& \left\|\left(I_{d}-P^{A}(t)\right) W(t, s)-R(t) Y(t, s)\right\| \leqslant\left\|\left(I_{d}-P^{A}(t)\right) W(t, s)-R(t) Y(t, s)\right\| \\
& \leqslant \int_{s}^{+\infty}\left\|X(t, \tau)\left(I_{d}-P^{A}(\tau)\right)\right\|_{s t}\|C(\tau)\|_{s}\left\|P^{B}(\tau) Y(\tau, s)\right\|_{s t} d \tau \\
& \quad+\int_{t}^{s}\left\|X(t, \tau)\left(I_{d}-P^{A}(\tau)\right)\right\|_{s t}\|C(\tau)\|_{s}\left\|\left(I_{n-d}-P^{B}(\tau)\right) Y(\tau, s)\right\|_{s t} d \tau \\
& \quad+\int_{0}^{t}\left\|X(t, \tau) P^{A}(\tau)\right\|_{s t}\|C(\tau)\|_{s}\left\|\left(I_{n-d}-P^{B}(\tau) Y(\tau, s)\right)\right\|_{s t} d \tau \\
& \leqslant k_{1} k_{2}\|C\|_{t}\left[\frac{e^{\beta_{1}(t-s)}}{\alpha_{2}+\beta_{1}}+\frac{e^{\beta_{1}(t-s)}-e^{\beta_{2}(t-s)}}{\beta_{2}-\beta_{1}}+\frac{e^{\beta_{2}(t-s)}}{\alpha_{1}+\beta_{2}}\right] \\
& \leqslant k_{1} k_{2}\|C\|_{t}\left[\frac{1}{\alpha_{2}+\beta_{1}}+\frac{1}{\left|\beta_{2}-\beta_{1}\right|}+\frac{1}{\alpha_{1}+\beta_{2}}\right] e^{\beta(t-s)},
\end{aligned}
$$

where exponents $\alpha_{1}, \alpha_{2}, \beta_{1}, \beta_{2}>0$ and constants $k_{1}, k_{2} \geqslant 1, \beta=\min \left\{\beta_{1} ; \beta_{2}\right\}$. If $\beta_{1}=\beta_{2}$, we have

$$
\int_{s}^{t} e^{\beta_{1}(t-\tau)} e^{\beta_{1}(\tau-s)} d \tau=(t-s) e^{\beta_{1}(t-s)} \leqslant c_{\beta} e^{\beta(t-s)}
$$

so that $\frac{1}{\left|\beta_{2}-\beta_{1}\right|}$ in the last inequality is replaced by $c_{\beta}$. Furthermore, note that if $t=s$, we do not have the first of the three terms in the inequality above so that we get

$$
\|R(t)\| \leqslant k_{1} k_{2}\|C\|_{t}\left[\frac{1}{\alpha_{2}+\beta_{1}}+\frac{1}{\alpha_{1}+\beta_{2}}\right] .
$$

The proof is complete.

Example 3.3. Consider the system

$$
\dot{x}=\left(\begin{array}{cc}
-1 & e^{-t} \\
0 & 1
\end{array}\right) x
$$

Both linear equations $\dot{x}=-2 x$ and $\dot{y}=-y$ have a nonuniform exponential dichotomy on $\mathbb{R}_{+}$with projection $\mathrm{P}^{\mathrm{A}}=\mathrm{P}^{\mathrm{B}}=1$. We show, with $\mathrm{C}(\mathrm{t})$ chosen like (3.9), equation (3.9) has a nonuniform exponential dichotomy on $\mathbb{R}_{+}$.

The fact is that we define the transition of (3.9) as

$$
T(t, s)=\left(\begin{array}{cc}
X(t, s) & W(t, s) \\
0 & Y(t, s)
\end{array}\right),
$$

when

$$
X(t, s)=e^{-2(t-s)}, \quad Y(t, s)=e^{-(t-s)}, \quad W(t, s)=\int_{s}^{t} X(t, \tau) C(\tau) Y(\tau, s) d \tau=e^{-2 t}-e^{-3 t+s} .
$$

In Theorem 2.3, we have

$$
L P^{B}=-\int_{0}^{+\infty}\left(I_{d}-P^{A}(\tau)\right) X(0, t) C(\tau) Y(\tau, 0) P^{B} d \tau=0
$$


then we define the projection of equation (3.9) as

$$
\mathrm{P}=\left(\begin{array}{cc}
\mathrm{P}^{\mathrm{A}} & \mathrm{LP}^{\mathrm{B}} \\
0 & \mathrm{P}^{\mathrm{B}}
\end{array}\right)
$$

and

$$
\mathrm{P}(\mathrm{t})=\mathrm{T}(\mathrm{t}, 0) \mathrm{PT}(0, \mathrm{t})=\left(\begin{array}{cc}
1 & \mathrm{e}^{-\mathrm{t}} \\
0 & 1
\end{array}\right)
$$

Then, $t \geqslant s \in J$, we consider

$$
\|\mathrm{T}(\mathrm{t}, \mathrm{s}) \mathrm{P}(\mathrm{s})\|=\left\|\left(\begin{array}{cc}
e^{-2(\mathrm{t}-\mathrm{s})} & \mathrm{e}^{-2 \mathrm{t}}-\mathrm{e}^{-3 \mathrm{t}+\mathrm{s}} \\
0 & \mathrm{e}^{-(\mathrm{t}-\mathrm{s})}
\end{array}\right)\left(\begin{array}{cc}
1 & e^{-\mathrm{t}} \\
0 & 1
\end{array}\right)\right\|=\left\|\left(\begin{array}{cc}
e^{-2(\mathrm{t}-\mathrm{s})} & \mathrm{e}^{-3 \mathrm{t}}-\mathrm{e}^{-4 \mathrm{t}+\mathrm{s}} \\
0 & \mathrm{e}^{-(\mathrm{t}-\mathrm{s})}
\end{array}\right)\right\| .
$$

We have

$$
\left\|e^{-3 t}-e^{-4 t+s}\right\| \leqslant e^{-3 t}+e^{-4 t+s} \leqslant 2 e^{-3 s} e^{-3(t-s)} .
$$

This means the equation (3.9) has a nonuniform exponential dichotomy on $\mathbb{R}_{+}$.

Example 3.4. We consider the equation

$$
\dot{x}=\left(\begin{array}{ccc}
-1 & e^{2 t} & C(t) \\
0 & 1 & \sin \ln (t+1)+\cos \ln (t+1)-\alpha
\end{array}\right) x,
$$

where

$$
A(t)=\left(\begin{array}{cc}
-1 & e^{2 t} \\
0 & 1
\end{array}\right), \quad B(t)=\sin \ln (t+1)+\cos \ln (t+1)-\alpha,
$$

and $C(t)$ is a matrix such that $C(t) \in C_{b}^{0}\left(J, \sup _{t \in J}\|\cdot\|_{t}\right)$.

Consider the equation

$$
\dot{\chi}=\left(\begin{array}{cc}
-1 & e^{2 t} \\
0 & 1
\end{array}\right) x
$$

The equation has the fundamental matrix solution as

$$
X(t)=\left(\begin{array}{cc}
e^{-t} & \left(e^{3 t}-e^{-t}\right) / 4 \\
0 & e^{t}
\end{array}\right)
$$

such that $\mathrm{X}(0)=\mathrm{I}$ and have an inverse matrix as

$$
X^{-1}(t)=\left(\begin{array}{cc}
e^{-t} & -\left(e^{3 t}-e^{-t}\right) / 4 \\
0 & e^{-t}
\end{array}\right) .
$$

With projection $\mathrm{P}^{\mathrm{A}}=\operatorname{diag}\{1,0\}$, we consider

$$
\left\|X(t) P^{A} X^{-1}(s)\right\|=\left\|\left(\begin{array}{cc}
e^{-(t-s)} & -\left(e^{-(t-s)+2 s}-e^{-t-s}\right) / 4 \\
0 & 0
\end{array}\right)\right\|=\frac{4+e^{2 s}-e^{-2 s}}{4} e^{-(t-s)}, \quad \forall t \geqslant s, \in \mathbb{R}_{+},
$$

and

$$
\left\|X(t)\left(I-P^{A}\right) X^{-1}(s)\right\|=\left\|\left(\begin{array}{cc}
0 & -\left(e^{-(s-t)+2 t}-e^{-t-s}\right) / 4 \\
0 & e^{-(s-t)}
\end{array}\right)\right\| \leqslant \frac{4+e^{2 s}-e^{-2 s}}{4} e^{-(s-t)}, \quad \forall t \leqslant s, \in \mathbb{R}_{+} .
$$

This means equation (3.11) has a nonuniform exponential dichotomy on $\mathbb{R}_{+}$with projection $\mathrm{P}^{\mathrm{A}}=$ $\operatorname{diag}\{1,0\}$. 
Next, we consider the equation

$$
\dot{\chi}=(\sin \ln (t+1)+\cos \ln (t+1)-\alpha) x, \quad t \in \mathbb{R}_{+} .
$$

When $1<\alpha<1+e^{-\frac{\pi}{2}}$, we have a fundamental solution matrix $X(t)$ such that $X(0)=1$ is

$$
X(t)=e^{(t+1) \sin \ln (+1)-\alpha t},
$$

hence

$$
\begin{aligned}
\left\|X(t) X^{-1}(s)\right\| & =e^{(t+1) \sin \ln (t+1)-\alpha t-(s+t) \sin \ln (s+1)+\alpha s} \\
& \leqslant e^{(s+1)(1-\sin \ln (s+1))} e^{-(\alpha-1)(t-s)}, \quad t \geqslant s, \in \mathbb{R}_{+} .
\end{aligned}
$$

Then, equation (3.12) has a nonuniform exponential dichotomy on $\mathbb{R}_{+}$. If not, with constant $\beta>0$ and $\mathrm{K}_{\beta}>0$, we have

$$
\left\|X(t) X^{-1}(s)\right\| \leqslant K_{\beta} e^{-\beta(t-s)}, \quad t \geqslant s, \in \mathbb{R}_{+} .
$$

With $s_{k}:=e^{(2 k-1 / 2) \pi}-1$ and $t_{k}:=e^{\pi} s_{k}-1$. Then, $\sin \ln \left(s_{k}+1\right)=-1, \sin \ln \left(t_{k}+1\right)=1$, and

$$
\left\|X\left(t_{k}\right) X^{-1}\left(s_{k}\right)\right\|=e^{\left(t_{k}+1\right) \sin \ln \left(t_{k}+1\right)-\alpha t_{k}-\left(s_{k}+t_{k}\right) \sin \ln \left(s_{k}+1\right)+\alpha s_{k}}=e^{\left(e^{\pi}+1\right) s_{k}-\alpha\left(e^{\pi}-1\right) s_{k}+\alpha+1} .
$$

Then, with $\beta>0,1<\alpha<1+e^{-\pi}$, we have

$$
e^{\left(e^{\pi}+1\right) s_{k}} \leqslant K_{\beta} e^{(\alpha-\beta)\left(e^{\pi}-1\right) s_{k}}<K_{\beta} e^{\left(1+e^{-\pi}\right)\left(e^{\pi}-1\right) s_{k}}=K_{\beta} e^{\left(e^{\pi}-e^{-\pi}\right) s_{k}} .
$$

This means $K_{\beta} \geqslant e^{\left(1+e^{-\pi}\right) s_{k}} \rightarrow+\infty, K \rightarrow+\infty$, contradict. Therefore, equation (3.12) has a nonuniform exponential dichotomy on $\mathbb{R}_{+}$.

According to Theorem 2.3, we have equation (3.10) which has a nonuniform exponential dichotomy on $\mathbb{R}_{+}$with projection $\mathrm{P}=\operatorname{diag}\{1,0,1\}$.

\section{Acknowledgment}

The first author was supported in part by the VNU Project of Vietnam National University No. QG15.01. The authors would like to extend their appreciations to members of the seminar "Dynamical Systems and Applications" at Hanoi University of Science for helpful suggestions and comments in preparing this paper.

\section{References}

[1] L. Barreira, D. Dragičević, C. Valls, Fredholm Operators and Nonuniform Exponential Dichotomies, Chaos Solitons Fractals, 85 (2016), 120-127. 1

[2] L. Barreira, D. Dragičević, C. Valls, Nonuniform exponential dichotomies and Fredholm operators for flows, Aequationes Math., 91 (2017), 301-316. 1

[3] L. Barreira, D. Dragičević, C. Valls, Nonuniform exponential dichotomies and Lyapunov functions, Regul. Chaotic Dyn., 22 (2017), 197-209. 1

[4] L. Barreira, Y. Pesin, Nonuniform Hyperbolicity. Dynamics of Systems with Nonzero Lyapunov Exponents, Encyclopedia of Mathematics and its Applications, Cambridge Univ. Press, Cambridge, (2007). 1

[5] L. Barreira, C. Valls, Stable manifolds for nonautonomous equations without exponential dichotomy, J. Differential Equations, 221 (2006), 58-90. 1

[6] L. Barreira, C. Valls, Stability of Nonautonomous Differential Equations, Springer, Berlin, (2008). 1

[7] L. Barreira, C. Valls, Nonuniform exponential dichotomies and admissibility, Discrete Contin. Dyn. Syst., 30 (2011), 39-53. 1

[8] L. Barreira, C. Valls, On two notions of exponential dichotomy, Dyn. Syst., 33 (2018), 708-721. 1

[9] F. Battelli, K. J. Palmer, Criteria for exponential dichotomy for triangular systems, J. Math. Anal. Appl., 428 (2015), 525-543. 2

[10] W. A. Coppel, Dichotomies in Stability Theory, Springer-Verlag, Berlin, (1978). 1

[11] O. Perron, Die Stabilitatsfrage bei Differentialgleichungen, (German) Math. Z., 32 (1930), 703-728. 1

[12] L. F. Zhou, K. N. Lu, W. N. Zhang, Equivalences between nonuniform exponential dichotomy and admissibility, J. Differential Equations, 262 (2017), 682-747. 1, 2 